APS

physics

This is the accepted manuscript made available via CHORUS. The article has been published as:

\title{
Experimental observation of electron-phonon coupling enhancement in Sn nanowires caused by phonon confinement effects
}

D. P. Lozano, S. Couet, C. Petermann, G. Hamoir, J. K. Jochum, T. Picot, E. Menéndez, K. Houben, V. Joly, V. A. Antohe, Michael Y. Hu, B. M. Leu, A. Alatas, Ayman H. Said, S. Roelants, B. Partoens, M. V. Milošević, F. M. Peeters, L. Piraux, J. Van de Vondel, A.

Vantomme, K. Temst, and M. J. Van Bael

Phys. Rev. B 99, 064512 - Published 21 February 2019

DOI: 10.1103/PhysRevB.99.064512 


\title{
Experimental observation of electron-phonon coupling enhancement in Sn nanowires caused by phonon confinement effects
}

\author{
D.P. Lozano, ${ }^{1}$ S. Couet, ${ }^{1}$ C. Petermann, ${ }^{1}$ G. Hamoir, ${ }^{2}$ J. K. Jochum,${ }^{3}$ T. Picot, ${ }^{3}$ E. Menéndez,${ }^{1,4}$ K. Houben,${ }^{3}$ V. \\ Joly, ${ }^{1}$ V.A. Antohe, ${ }^{2,5}$ Michael Y. Hu, ${ }^{6}$ B. M. Leu, ${ }^{6,7}$ A. Alatas, ${ }^{6}$ Ayman H. Said,${ }^{6}$ S. Roelants, ${ }^{8}$ B. Partoens, ${ }^{8}$ M. \\ V. Milošević, ${ }^{8}$ F. Peeters ${ }^{8}$ L. Piraux,${ }^{2}$ J. Van de Vondel,${ }^{3}$ A. Vantomme, ${ }^{1}$ K. Temst,${ }^{1}$ and M. J. Van Bael ${ }^{3}$ \\ ${ }^{1}$ KU Leuven, Instituut voor Kern-en Stralingsfysica, Celestijnenlaan 200 D, 3001 Leuven, Belgium. \\ ${ }^{2}$ Institute of Condensed Matter and Nanosciences (IMCN), \\ Université catholique de Louvain, Place Croix du Sud 1, 1348 Louvain-la-Neuve, Belgium. \\ ${ }^{3}$ Laboratory of Solid-State Physics and Magnetism, \\ KU Leuven, Celestijnenlaan 200D, 3001 Leuven, Belgium \\ ${ }^{4}$ Departament de Física, Universitat Autònoma de Barcelona, E-08193 Cerdanyola del Vallès, Spain. \\ ${ }^{5}$ Research and Development Center for Materials and Electronic \& Optoelectronic Devices (MDEO), \\ Faculty of Physics, University of Bucharest, Atomistilor str. 405, 077125 Bucharest-Magurele, Romania. \\ ${ }^{6}$ Advanced Photon Source, Argonne National Laboratory, Argonne, Illinois 60439, USA. \\ ${ }^{7}$ Miami University, Department of Physics, Oxford, Ohio 45056, USA. \\ ${ }^{8}$ Departement Fysica, Universiteit Antwerpen, Groenenborgerlaan 171, B-2020 Antwerpen, Belgium.
}

\begin{abstract}
Reducing the size of a superconductor below its characteristic lengths scales can either enhance or suppress its critical temperature $\left(\mathrm{T}_{c}\right)$. Depending on the bulk value of the electron-phonon coupling strength, electronic and phonon confinement effects will play different roles in the modification of $\mathrm{T}_{c}$. Experimentally disentangling each contribution has remained a challenge. We have measured both the phonon density of states and $\mathrm{T}_{c}$ of $\mathrm{Sn}$ nanowires with diameters of $18 \mathrm{~nm}, 35 \mathrm{~nm}$ and $100 \mathrm{~nm}$ in order to quantify the effects of phonon confinement on superconductivity. We observe a shift of the phonon frequency towards the low energy region and an increase in the electron-phonon coupling constant that can account for the measured increase in $\mathrm{T}_{c}$.
\end{abstract}

\section{INTRODUCTION}

The electron-phonon interaction plays a fundamental role in electrical transport [1], thermal conductivity [2] and many-body phenomena such as superconductivity [3]. In reduced dimensions, the strength of this interaction is modified due to size effects in both, electronic levels and phonon spectrum. For instance, nanostructuring superconductors leads to dramatic changes in their properties compared to their bulk counterparts. These changes appear mostly once the size of the superconductor becomes smaller than the coherence length $\xi_{0}$ resulting in a modification of the critical temperature $\mathrm{T}_{c}[4]$ the critical magnetic field $\mathrm{H}_{c}[5,6]$ and the superconducting gap $\Delta_{0}[7]$. Particularly, the behavior of $\mathrm{T}_{c}$ has received a lot of interest since its modification depends on how nanostructuring affects the electron-phonon interaction (e-ph), reflected in the value of the electron-phonon coupling constant $\lambda_{e-p h}[8]$.

Nanostructured weak-coupling superconductors show a progressive enhancement of $\mathrm{T}_{c}$ with a decrease of the characteristic dimension of the system. An increase of $\mathrm{T}_{c}$ of about $25 \%$ with respect to bulk $\mathrm{Al}$ has been observed in Al nanowires with $8 \mathrm{~nm}$ diameter [9], while in Sn nanowires with $20 \mathrm{~nm}$ diameter the enhancement was only about $10 \%$ [10]. For In nanoparticles with $36 \mathrm{~nm}$ diameter, the $\mathrm{T}_{c}$ increase was smaller, only about $5 \%$ [11]. This $\mathrm{T}_{c}$ enhancement has generally been attributed to an enhancement of the electron-phonon coupling caused by surface phonon modes and crystallite boundaries [12]. On the other hand, $\mathrm{T}_{c}$ of strong-coupling materials seems not to be severely affected by dimensional effects. The
$\mathrm{T}_{c}$ of $\mathrm{Pb}$ nanoparticles decreases slightly within a diameter range spanning from $65 \mathrm{~nm}$ down to $7 \mathrm{~nm}$ [13], after which $\mathrm{T}_{c}$ drops abruptly. In the case of $\mathrm{Nb}$, a progressive decrease of $\mathrm{T}_{c}$ was observed in nanowires with diameter between $420 \mathrm{~nm}$ and $80 \mathrm{~nm}$ [14]. However, these materials are equally affected by surface and boundary effects as weak-coupling superconductors are, since those are a consequence of the sample's geometry. This has been demonstrated in $\mathrm{Pb}$ nanoparticles embedded into nanoporous glass [15]. Therefore, a mechanism that opposes the enhancement of $\lambda_{e-p h}$ should be present.

There exist two effects that play an important role in the $\mathrm{T}_{c}$ modification of nanoscale superconductors. First, oscillations of the mean electron density near the Fermi level as a consequence of the discretization of the energy levels in reduced dimensions, known as quantum size effects (QSE) [16], and second, the shift of phonon modes towards low energies as a consequence of phonon confinement, known as phonon softening [17]. Phonon softening is expected to increase $\mathrm{T}_{c}$, while the influence of QSE depends on the electron-phonon coupling strength. Even a combination of these two effects can explain the observed $\mathrm{T}_{c}$ behavior. Phonon softening has been observed in $\mathrm{Sn}$ and $\mathrm{Pb}$ nanoparticles embedded into porous glass $[15,18]$. However, no link with the effect on $\mathrm{T}_{c}$ was established. Similar measurements were carried out in $\mathrm{Nb}_{3} \mathrm{Sn}$ thin films [19], where the role of phonon softening was quantified, revealing that its contribution was not enough to explain the change of $\mathrm{T}_{c}$. QSE were pointed out as the main effect responsible for this change.

Disentangling and quantifying the individual effects of phonon softening and QSE remains a challenge, mainly 
due to the lack of techniques capable of measuring phonons in nanoscale superconductors. Electron tunneling experiments in superconducting junctions [20] are able to provide information about the electron-phonon interaction; however, they are intrinsically influenced by electronic effects. Therefore, techniques that allow for the differentiation between these two effects are required.

In this work, we quantify the contribution of phonon softening effects to the modification of $\mathrm{T}_{c}$ in nanoscale superconductors. Measuring the phonon density of states $\mathrm{F}(\mathrm{E})$ of $\mathrm{Sn}$ nanowire arrays embedded in porous alumina $\left(\mathrm{Al}_{2} \mathrm{O}_{3}\right)$ matrices with different pore diameters using nuclear resonant inelastic X-ray scattering (NRIXS) measurements allows us to determine the shifting of the phonon frequencies and the increment of the electronphonon coupling upon decreasing diameter. The combination of these results with data from transport measurements suggests that phonon softening is responsible for the $\mathrm{T}_{c}$ enhancement observed in the $\mathrm{Sn}$ nanowire samples studied.

\section{SAMPLE PREPARATION AND STRUCTURAL CHARACTERIZATION}

Tin nanowire arrays were synthesized by electrodepositing $\mathrm{Sn}$ from an aqueous electrolyte containing $7 \mathrm{~g} / \mathrm{L}$ $\mathrm{C}_{4} \mathrm{H}_{6} \mathrm{O}_{6}, 115 \mathrm{~g} / \mathrm{L} \mathrm{Na}{ }_{4} \mathrm{P}_{2} \mathrm{O}_{7} \cdot 10 \mathrm{H}_{2} \mathrm{O}, 30 \mathrm{~g} / \mathrm{L}{ }^{119} \mathrm{SnCl}_{2}$ - $2 \mathrm{H}_{2} \mathrm{O}$ and $0.4 \mathrm{~g} / \mathrm{L}$ gelatin [21] into $50 \mu$ m-thick nanoporous $\mathrm{Al}_{2} \mathrm{O}_{3}$ membranes with pore diameters of $18 \pm 3 \mathrm{~nm}, 35 \pm 3 \mathrm{~nm}$ and $100 \pm 10 \mathrm{~nm}$. A bi-layer consisting of $10 \mathrm{~nm}$ of $\mathrm{Cr}$ followed by $300 \mathrm{~nm}$ of $\mathrm{Au}$ was deposited by e-beam evaporation on one side of the membranes to serve as working electrode. Isotopically enriched ${ }^{119} \mathrm{SnCl}_{2} \cdot 2 \mathrm{H}_{2} \mathrm{O}$ was developed in house due to the lack of its commercial availability. The electrochemical growth of homogenous nanowires within the $\mathrm{Al}_{2} \mathrm{O}_{3}$ membranes was carried out in a three-electrode potentiostatic configuration by applying to the working electrode $-0.9 \mathrm{~V}$ versus a double-junction $\mathrm{Ag} / \mathrm{AgCl}$ reference electrode ( $\mathrm{KCl}$ saturated, $\mathrm{E}=0.197 \mathrm{~V}$ ), while a $\mathrm{Pt}$ foil was installed in the bath as a counter electrode. The process was stopped once the Sn overfilled the membrane. In order to check the reproducibility of the critical temperature, two samples with diameters of $35 \mathrm{~nm}$ (samples $\mathrm{c}$ and $\mathrm{d}$ ) and two samples with diameters of $100 \mathrm{~nm}$ (samples $\mathrm{g}$ and $\mathrm{h}$ ) were prepared using this method. Due to proximity effects the top and/or bottom layer can significantly affect the $T_{c}$ of the nanowires [22]. To identify the importance of this effect, nanowires overgrown with $\mathrm{Cu}$ were fabricated as well. For these samples the growth within the membranes was stopped once the wires reached $25 \mu \mathrm{m}$ and then $\mathrm{Cu}$ was electrodeposited to fill the rest of the template. Samples with diameters of $35 \mathrm{~nm}$ (samples e and $\mathrm{f}$ ) and $18 \mathrm{~nm}$ (samples a and b) were prepared using this method. After the growth process the membranes where taken out of the electrolyte in order to characterize the samples. Additionally, three more samples with nanowire diameters of $18 \mathrm{~nm}, 35 \mathrm{~nm}$ and $100 \mathrm{~nm}$ were grown using the Mössbauer isotope ${ }^{119} \mathrm{Sn}$ in order to perform the NRIXS measurements. The latter set of samples was not overgrown in order to avoid the contribution of unconfined nanowire caps on the template surface to the NRIXS measurements. As shown in the cross-sectional Scanning Electron Microscope (SEM) image in Fig. 1 a), the samples are formed by an array of vertically aligned nanowires. The diameter of the nanowires slightly fluctuates along the longitudinal direction, its standard deviations are $6 \mathrm{~nm}, 2$ $\mathrm{nm}$ and $1.5 \mathrm{~nm}$ for the $100 \mathrm{~nm}, 35 \mathrm{~nm}$ and $18 \mathrm{~nm}$ samples.

Structural characterization was performed by $\theta / 2 \theta$ $\mathrm{X}$-ray diffraction (XRD) and grazing incidence XRD (GIXRD) with an incidence angle of $4^{\circ}$, using $\mathrm{Cu} \mathrm{K} \mathrm{K}_{\alpha 1}$ radiation. Figure $1 \mathrm{~b}$ ) shows the XRD patterns for the three types of samples investigated. In all samples, three peaks corresponding to $\beta$-Sn can be observed at approximately $30^{\circ}, 56^{\circ}$ and $63^{\circ}$. In addition, an $\mathrm{Au}$ peak arises from the cathode at $38^{\circ}$. The $\theta / 2 \theta$ XRD patterns of all samples are consistent with a polycrystalline array of nanowires containing a highly textured $\beta$-Sn phase oriented along (200) planes with traces of (301) oriented planes. GIXRD measurements (available in the supplemental material) show either no peaks or tiny traces of the (200) $\beta$-Sn peak just above the background level, confirming the strong texture of the $\beta$-Sn nanowires. To obtain the (average coherently diffracting) crystallite size of the $\beta$-Sn phase along the direction of the wires, Rietveld refinement [23] of the $\theta / 2 \theta$ XRD patterns was performed using the MAUD software [24]. The grain size of the $35 \mathrm{~nm}$ and $100 \mathrm{~nm}$ diameter samples is larger than $200 \mathrm{~nm}$ and cannot be resolved by line profile analysis [25], while a grain size of $185 \mathrm{~nm}$ is found for the $18 \mathrm{~nm}$ diameter sample, indicating that the nanowires consist of a series of strongly elongated grains along their axis.

\section{SUPERCONDUCTING PROPERTIES}

Transport measurements were performed by making four contacts on the sample, two on the top surface and two at the bottom. A schematic drawing of the contact geometry is shown in the inset of Fig. 2. The normalized resistance $\mathrm{R}(\mathrm{T}) / \mathrm{R}(4.5 \mathrm{~K})$ of three representative samples (one of each thickness) as a function of temperature are displayed in Fig. 2, showing a distinct number of features. Marked with black arrows, a first resistance drop occurs around $4 \mathrm{~K}$. Beyond this transition and towards lower temperatures the resistance does not drop to zero, but it slowly decreases until a second transition is visible in a temperature rage around $2.25 \mathrm{~K}$. After this transition the zero resistance state is still not reached. The normalized resistance curves of the other samples can be found in the supplementary information. In general, for 

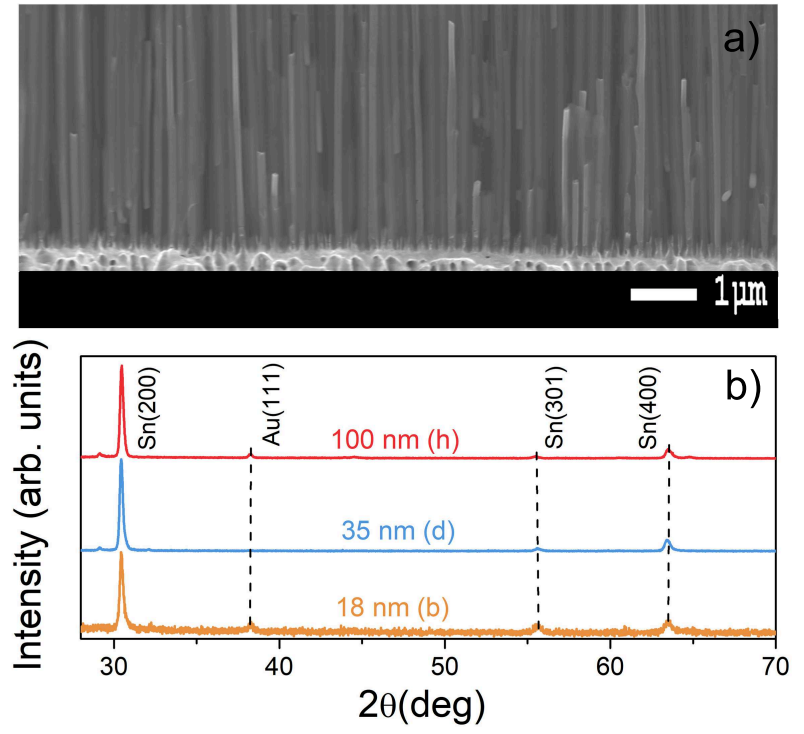

FIG. 1. a) SEM image of a $50 \mu \mathrm{m}$ membrane containing Sn nanowires with a diameter of $100 \mathrm{~nm}$. b) XRD spectra for the $\mathrm{Sn}$ nanowires embedded in $\mathrm{Al}_{2} \mathrm{O}_{3}$ porous matrices with different pore diameter.

each nanowire diameter the obtained transitions display a very similar behavior.

We attribute the first resistance drop to the superconducting transition of the strongly elongated grains forming the nanowires. In this viewpoint, $\mathrm{T}_{c}$ is defined as the middle point of this transition, thus obtaining values of $4.05 \pm 0.05 \mathrm{~K}, 3.95 \pm 0.05 \mathrm{~K}$ and $3.82 \pm 0.05 \mathrm{~K}$ for the 18 $\mathrm{nm}, 35 \mathrm{~nm}$ and $100 \mathrm{~nm}$, respectively. Moreover, the comparison between $\mathrm{Sn}$ overgrown and Cu overgrown samples directly shows that the overgrown layer does not impact the obtained $\mathrm{T}_{c}$. This represents an enhancement of up to $10 \%$ compared to $3.72 \mathrm{~K}$, the bulk $\mathrm{T}_{c}$ value. These values of $T_{c}$ are in agreement with the values observed in other Sn nanowire systems [10, 27, 28]. The second resistance drop occurs in a temperature range between $2.0 \mathrm{~K}$ and $2.5 \mathrm{~K}$. While the first superconducting transition is related to the local superconductivity within the narrow elongated grains of the individual nanowires; we hypothesize that the second transition is related to the emergence of long-range superconductivity when coupling between these grains is established at lower temperatures. This feature will be the topic of future work.

In addition to the two resistance drops observed, a third one would be expected around $3.7 \mathrm{~K}$ in the Sn overgrown samples due to the superconducting transition of the bulk overgrown layer. However, such a transition only appears in the magnetoresistance curve of one of the Sn overgrown $35 \mathrm{~nm}$ samples (sample c), shown in the Supplemental material [26], and not in the resistance versus temperature curve in Fig. 2. Figure S3 in the supplemental material shows a narrow transition appearing already at $3.7 \mathrm{~K}$ and visible in the entire temperature range explored. It can be suppressed by applying a magnetic field

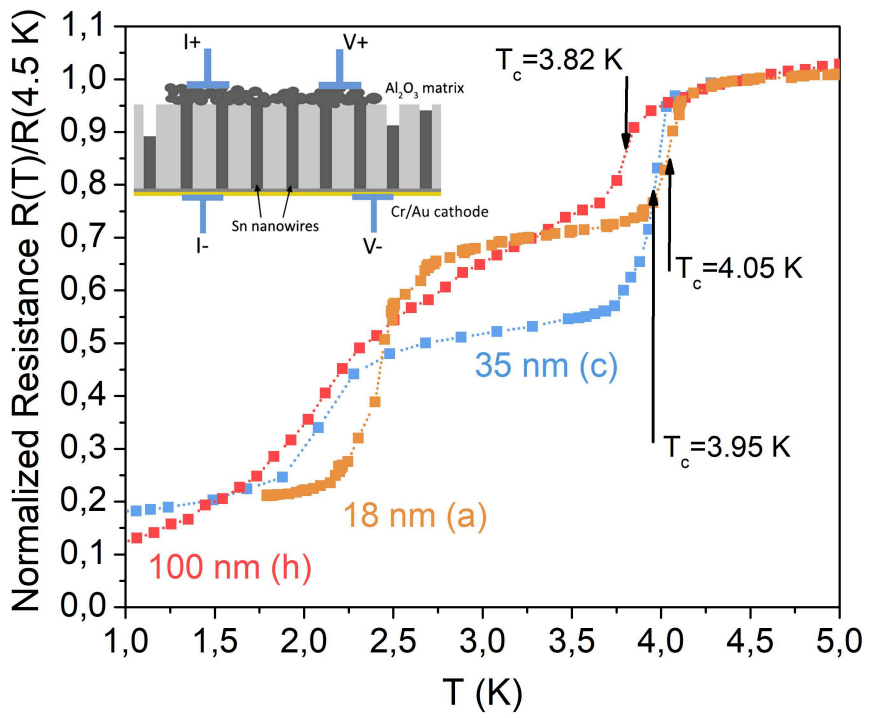

FIG. 2. Normalized resistance measurement of the Sn nanowires. The diameters of the nanowires are $100 \mathrm{~nm}(\mathrm{~h}$, red), $35 \mathrm{~nm}$ (c, blue), and $18 \mathrm{~nm}$ (a, orange). The black arrows indicate the critical temperature defined according to the criterion explained in the text. The inset depicts a sketch of the measurement configuration.

of about $0.05 \mathrm{~T}$ at $1 \mathrm{~K}$. This value is comparable to the critical magnetic field of bulk Sn [29], therefore, this transition is likely caused by the bulk Sn layer. The $100 \mathrm{~nm}$ and one of the $35 \mathrm{~nm}$ Sn overgrown samples (samples $\mathrm{g}, \mathrm{h}$ and d) present only two transitions. One possible explanation for the absence of the superconducting transition in the overgrown layer could be that the large contact area of this layer contributed to a very small fraction of the system's total resistance. Then, the decrease in resistance caused by the superconducting transition of the overgrown layer would be too small to be discernible in our data. The noise level in the $100 \mathrm{~nm}$ samples is $1 \mathrm{mV}$ while it is $0.5 \mathrm{mV}$ in the $35 \mathrm{~nm}$ sample (sample d). On the other hand, the resistance drop caused by the overgrown layer in the $35 \mathrm{~nm}$ sample (sample c) is only 0.15 $\mathrm{mV}$. Therefore the effect of the overgrown layer's superconducting transition is hidden by the noise in samples $\mathrm{g}$, $\mathrm{h}$ and d. Tian et al. studied Sn nanowires with diameter ranging from $20 \mathrm{~nm}$ to $160 \mathrm{~nm}$ embedded in porous matrices contacted by bulk Sn electrodes [10, 30]. Similar to our resistance versus temperature data, their data do not show any evidence of the superconducting transition in the bulk Sn electrodes.

The superconducting phase boundaries can provide additional evidence to our interpretation of the first resistance drop, since strong dimensional effects are expected in their temperature dependence due to the confined geometry of the grains forming the nanowires. The superconducting phase boundaries were determined measuring the magnetoresistance of the nanowires at different temperatures while an external magnetic field was applied 
perpendicular to the nanowires axis (see Supplemental material [26]). The magnetic field corresponding to $90 \%$ of the resistance between the normal and the lowest resistance state was used as criterion for $\mathrm{H}_{c}$. This criterion is used to guarantee that the phase boundaries are determined using values of the resistance similar to those used for determining the $\mathrm{T}_{c}$ of the first transition. Results are shown in Fig. 3. The superconducting region significantly increases as the diameter is reduced. The solid lines are fittings to the experimental data using Eq. $1[31]$

$$
H_{c}(T)=\frac{\sqrt{3} \phi_{0}}{\pi d \mu_{0} \xi_{G L}(0)} \sqrt{1-\frac{T}{T_{c}}}
$$

where $\phi_{0}$ is the magnetic flux quantum, $\mathrm{d}$ is the diameter of the nanowires and $\xi_{G L}(0)$ is the Ginzburg-Landau coherence length at $0 \mathrm{~K}$. This last one was used as a fitting parameter. There is a good correspondence between Eq. 1 and the experimental data, especially at temperatures close to $T_{c}$. Note that this relationship between $\mathrm{H}_{c}(\mathrm{~T})$ and $\mathrm{T}$ is typically observed in narrow superconducting lines [32], rather than the characteristic linear relationship observed in bulk superconductors [33], as we had anticipated, and confirms that the first resistance drop and consequently $\mathrm{T}_{c}$ corresponds to the nanowires. This, together with the strong diameter dependence of the critical field confirms that the critical field probed corresponds to the nanowires.

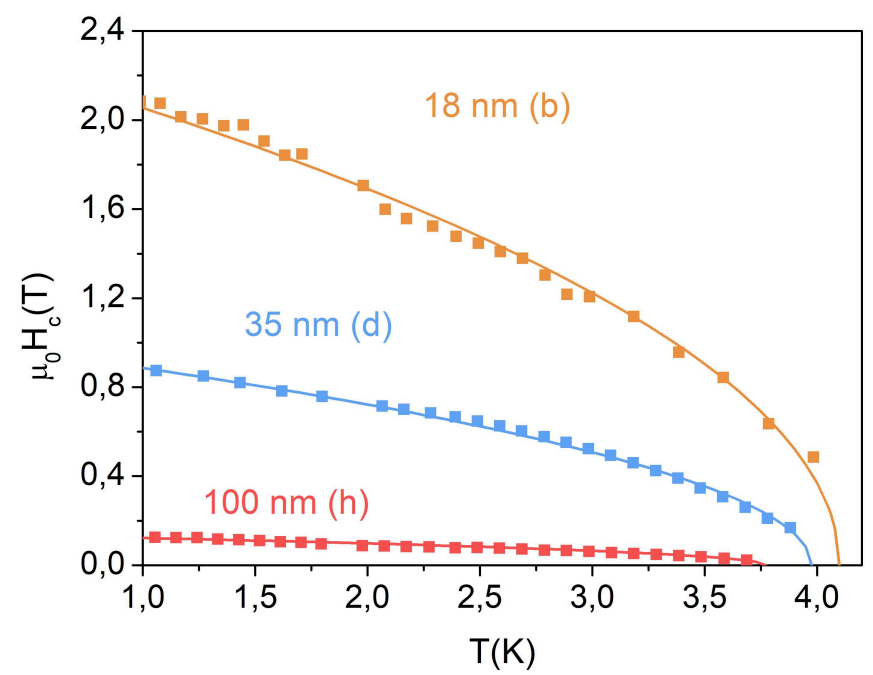

FIG. 3. Superconducting phase boundaries for the $18 \mathrm{~nm}$ (b, orange), $35 \mathrm{~nm}$ (d, blue) and $100 \mathrm{~nm}$ (h, red) samples, when a magnetic field is applied perpendicular to the nanowires axis.

The $\xi_{G L}(0)$ values obtained from the phase boundary fittings are shown in Fig. 4. Note that Eq. 1 strictly applies to a narrow superconducting line of rectangular cross-section and thickness $d$ in a perpendicular magnetic field [32]. The $\xi_{G L}(0)$ values should be considered an estimation to the coherence length, where the diameter of the wire plays the role of the line thickness. $\xi_{G L}(0)$ is significantly smaller than $\xi_{G L}(0)$ for bulk Sn [10], and it decreases monotonically as the nanowire diameter is reduced. This suggests that our nanowires are in the dirty limit since $\xi_{G L}(0)$ is expected to decrease in nanostructured dirty superconductors [34] with decreasing particle size as a consequence of the reduction in the effective mean free path [35].

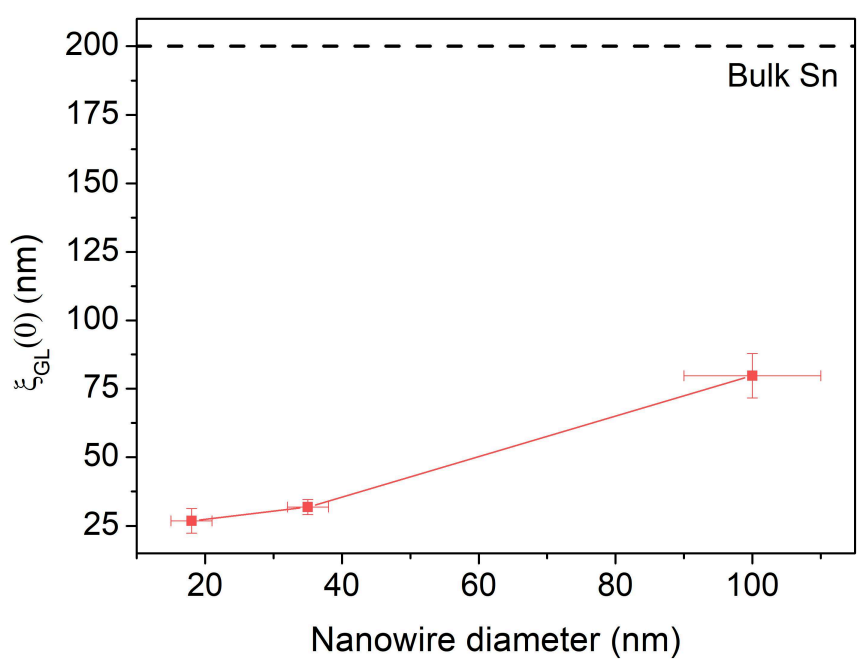

FIG. 4. Coherence length (red squares) of the $18 \mathrm{~nm}, 35$ $\mathrm{nm}$ and $100 \mathrm{~nm}$ samples when a magnetic field is applied perpendicular to their longitudinal axis compared to the bulk value (black dashed line). The red line is a guide to the eye.

\section{PHONON DENSITY OF STATES}

The phonon density of states of the nanowires was measured using NRIXS [36]. This technique allows the extraction of $\mathrm{F}(\mathrm{E})$ corresponding to only the Mössbaueractive material, ${ }^{119} \mathrm{Sn}$ in this case. No contributions from other atoms are measured, unlike inelastic neutron scattering (INS) or inelastic X-ray scattering (IXS). Moreover, the determination of $\mathrm{F}(\mathrm{E})$ from the NRIXS is model independent and it has been successfully applied to other nanosystems [37]. Measurements were performed at sector 30 of the Advanced Photon Source (APS). Details of the experimental set up are reported elsewhere [38]. The measured spectra consist of the photon count rate as a function of the energy difference between the incident photon and the Mössbauer nuclear transition in ${ }^{119} \mathrm{Sn}$. The set of samples used in the measurement are $99 \%$ isotopically enriched ${ }^{119} \mathrm{Sn}$ nanowires, not overgrown. Therefore, the recorded spectra correspond uniquely to the nanowires. The NRIXS scans were taken in two different configurations: the X-ray beam parallel and perpendicular to the nanowires axis. The spectra were converted to $\mathrm{F}(\mathrm{E})$ following the procedure described by Sturhahn [39] and using the PHOENIX software [40]. For each configuration, the partial $\mathrm{F}(\mathrm{E})$ was derived (see sup- 
plemental material [26]) and summed according to the following ratio: $\frac{2}{3} \mathrm{~F}(\mathrm{E})_{\text {perpendicular }}+\frac{1}{3} \mathrm{~F}(\mathrm{E})_{\text {parallel }}$ [41], in order to obtain the complete $\mathrm{F}(\mathrm{E})$ of the sample.

Figure 5 shows the $\mathrm{F}(\mathrm{E})$ 's obtained for the different samples together with the bulk Sn F(E) calculated using density functional theory (DFT) and convoluted with the experimental resolution function. All of them are compared to the $\mathrm{F}(\mathrm{E})$ of a $\mathrm{Sn}$ foil. The calculated $\mathrm{F}(\mathrm{E})$ is in good agreement with the Sn foil $\mathrm{F}(\mathrm{E})$, especially in the low and high energy parts; even the Sn cut off energy is accurately predicted. A number of significant differences are observed when comparing the phonon spectra of the nanowires and bulk Sn. The intensity of the high energy phonon modes at $15 \mathrm{meV}$ and $16.5 \mathrm{meV}$ is noticeably lower for the three diameters studied. Furthermore, the peak structure is completely broadened in the $100 \mathrm{~nm}$ and $18 \mathrm{~nm} \mathrm{~F}(\mathrm{E})$. In the medium energy region, between 7 - $14 \mathrm{meV}$, an increase of the phonon modes occurs, especially in the $18 \mathrm{~nm}$ sample. Moreover, the intensity of the low energy modes, between $0-3 \mathrm{meV}$, is also enhanced. This feature is better appreciated in Fig. 6, which shows how this enhancement is more pronounced as the diameter of the wires decreases. On the other hand, the intensity of the peak at $5 \mathrm{meV}$ is considerably reduced. Finally, a number of modes extending beyond the cut-off energy $18.5 \mathrm{meV}$ are visible. These differences observed between the Sn foil F(E) and the Sn nanowires $\mathrm{F}(\mathrm{E})$ are caused by the structural and morphological differences between the different samples.

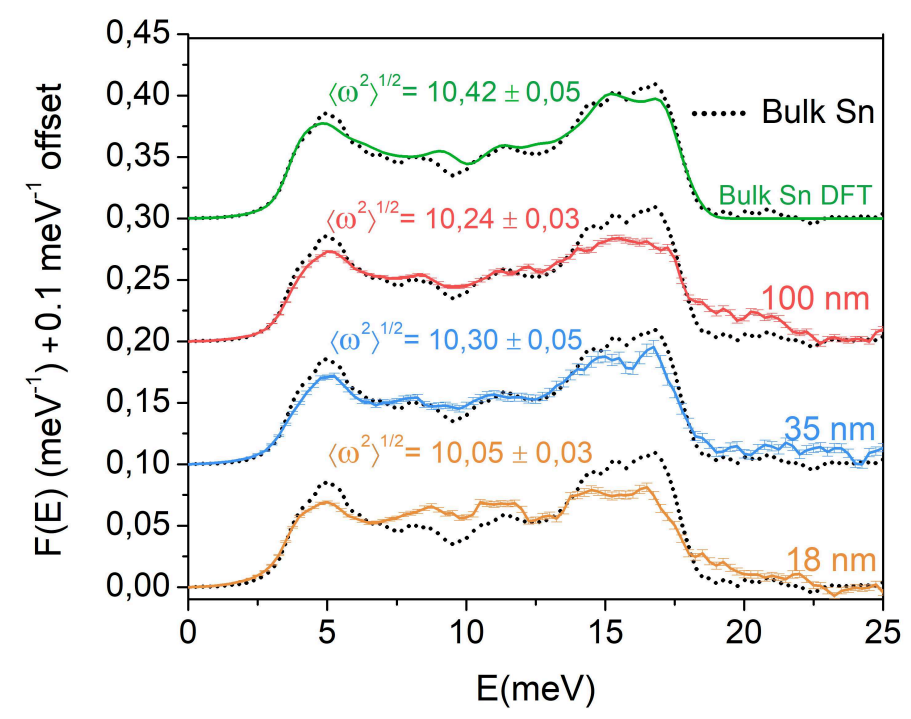

FIG. 5. Phonon density of states of bulk Sn calculated using DFT methods (green) and of membranes containing nanowires of $100 \mathrm{~nm}, 35 \mathrm{~nm}$ and $18 \mathrm{~nm}$ diameter (red, blue and orange, respectively) taken at $20 \mathrm{~K}$. The black dotted curve represents the $\mathrm{F}(\mathrm{E})$ of a bulk Sn foil measured during a previous experiment [42].

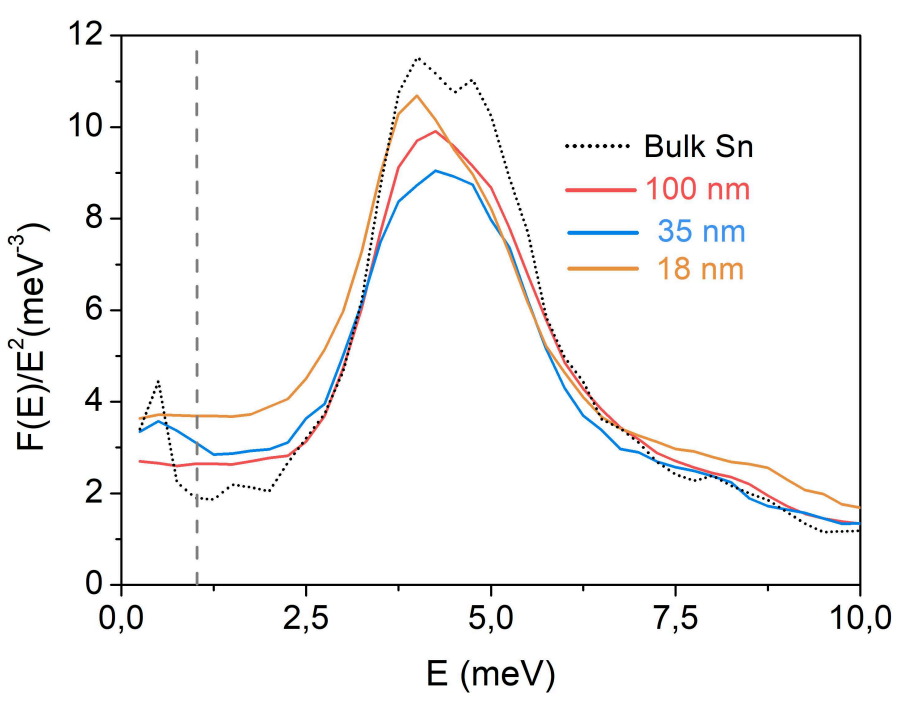

FIG. 6. Reduced phonon density of states $\left(\mathrm{F}(\mathrm{E}) / \mathrm{E}^{2}\right)$ over an energy region from $0 \mathrm{meV}$ to $10 \mathrm{meV}$, of Sn nanowires with diameters of $100 \mathrm{~nm}, 35 \mathrm{~nm}$ and $18 \mathrm{~nm}$ diameter (red, blue and orange, respectively), a bulk $\mathrm{Sn}$ foil taken at $20 \mathrm{~K}$. The grey dashed line indicates the full width at half maximum of the experimental resolution function

\section{DISCUSSION}

Using the measured $\mathrm{F}(\mathrm{E})$ 's it is possible to calculate how the changes in $\mathrm{F}(\mathrm{E})$ with respect to bulk affect $\mathrm{T}_{c}$. The superconducting critical temperature can be calculated using the Allen-Dynes equation [43], which is a correction of the McMillan expression for the $\mathrm{T}_{c}$ of conventional superconductors.

$$
T_{c}=\frac{f_{1} f_{2} \omega_{l o g}}{1.2} e^{-\frac{1.04\left(1+\lambda_{e-p h}\right)}{\lambda_{e-p h}-\mu^{*}-0.62 \lambda_{e-p h} \mu^{*}}}
$$

where the electron-phonon coupling constant is calculated as

$$
\lambda_{e-p h}=2 \int \frac{\alpha^{2}(E) F(E)}{E} d E
$$

the logarithmic frequency average is

$$
\omega_{l o g}=e^{\frac{2}{\lambda_{e}-p h} \int \frac{\alpha^{2}(E) F(E)}{E} \log (E) d E}
$$

and the prefactors, that depend on the characteristic phonon frequency, are

$$
\begin{gathered}
f_{1}=\left(1+\left(\frac{\lambda_{e-p h}}{2.46\left(1+3.8 \mu^{*}\right)}\right)\right)^{1 / 3} \\
f_{2}=1+\frac{\lambda_{e-p h}^{2}\left(\frac{\left\langle\omega^{2}\right\rangle^{1 / 2}}{\omega_{l o g}}-1\right)}{\lambda_{e-p h}^{2}+\left(1.82\left(1+6.3 \mu^{*} \frac{\left\langle\omega^{2}\right\rangle^{1 / 2}}{\omega_{l o g}}\right)\right)} \\
\left\langle\omega^{2}\right\rangle=\left(\frac{2}{\lambda_{e-p h}} \int \alpha^{2}(E) F(E) E d E\right)
\end{gathered}
$$


The electron-phonon coupling strength function $\alpha^{2}(\mathrm{E})$, shown in the supplemental material [26], has been calculated from the ratio between the Eliashberg function $\alpha^{2}(\mathrm{E}) \mathrm{F}(\mathrm{E})$ determined from electron tunneling measurements [20] and F(E) of a Sn foil that we previously measured [42]. This method has also been successfully applied in order to estimate the form of $\alpha^{2}(\mathrm{E})$ in $\mathrm{Nb}_{3} \mathrm{Sn}$ thin films [19]. The value of $\mu^{*}$ is fixed to 0.107 and is chosen to yield the bulk $\mathrm{T}_{c}$ value $3.7 \mathrm{~K}$ when the bulk $\mathrm{Sn}$ $\mathrm{F}(\mathrm{E})$ is used in the calculation. The chosen value of $\mu^{*}$ agrees with the values found in literature [44]. The same $\mu^{*}$ and $\alpha^{2}(\mathrm{E})$ were subsequently used to calculate $\mathrm{T}_{c}$ for the nanowires. Values of $4.20 \pm 0.03 \mathrm{~K}, 3.93 \pm 0.04 \mathrm{~K}$ and $3.87 \pm 0.03 \mathrm{~K}$ are found for the $18 \mathrm{~nm}, 35 \mathrm{~nm}$ and 100 nm nanowires, respectively. Figure 7 shows a comparison between $\mathrm{T}_{c}$ obtained from the transport measurements, $\mathrm{T}_{c}$ obtained using the experimentally derived $\mathrm{F}(\mathrm{E})$, and the bulk Sn $\mathrm{T}_{c}$. The values of $\mathrm{T}_{c}$ for the $100 \mathrm{~nm}$ and 35 $\mathrm{nm}$ samples obtained in the transport measurements are in agreement with the values calculated using the phonon density of states. In the case of the $18 \mathrm{~nm}$ nanowires a deviation occurs.

The value of the electron-phonon coupling parameter $\lambda_{e-p h}$ is displayed in the inset of Fig. 7. Similarly to the trend observed in $\mathrm{T}_{c}, \lambda_{e-p h}$ increases as the diameter is reduced. More specifically, an increase between $2.5 \%$ and $4.7 \%$ with respect to bulk $\mathrm{Sn}$ is obtained. This enhancement originates from the phonon softening effects. Calculations of the characteristic phonon frequency $\left\langle\omega^{2}\right\rangle^{1 / 2}$, shown in Fig. 5, indicate that there is a shift of the phonon frequencies towards the low energy region. Low energy phonons have a bigger impact on $\lambda_{e-p h}$ than high energy phonons due to the fact that the spectrum $\alpha^{2}(\mathrm{E}) \mathrm{F}(\mathrm{E})$ is weighted by a factor $1 / \mathrm{E}$. Phonon softening specially affects nanoscale materials because the ratio of surface to volume atoms is higher than in bulk. These surface atoms are characterized for having lower vibrational frequencies than the bulk ones, known as surface modes $[45,46]$, which shifts the phonon spectrum to the low energy region. While $\mathrm{T}_{c}$ and $\lambda_{e-p h}$ increase monotonically as the diameter is reduced, it is not the case for $\left\langle\omega^{2}\right\rangle^{1 / 2}$. The discrepancy arises from the shape of $\mathrm{F}(\mathrm{E})$ at high energies. As can be seen in Fig. 5, the $\mathrm{F}(\mathrm{E})$ shape of the $35 \mathrm{~nm}$ sample around $15 \mathrm{meV}$ resembles much more bulk Sn than in the case of the $100 \mathrm{~nm}$ nanowires. The contribution of these phonon modes to $\left\langle\omega^{2}\right\rangle^{1 / 2}$ is more relevant than the low energy ones since, as equation 5 shows, the spectrum $\alpha^{2}(\mathrm{E}) \mathrm{F}(\mathrm{E})$ is weighted by a factor $\mathrm{E}$. Nevertheless, $\mathrm{T}_{c}$ depends on more quantities apart from $\left\langle\omega^{2}\right\rangle^{1 / 2}$. A small deviation in one of the parameters can be compensated by the others, resulting in a $\mathrm{T}_{c}$ enhancement.

Despite the good correspondence between the $\mathrm{T}_{c}$ trend obtained from transport measurements and the one calculated using the phonon spectra, the correspondence between $\mathrm{T}_{c, \text { transport }}$ and $\mathrm{T}_{c, \text { phonons }}$ of the $18 \mathrm{~nm}$ sample is less good. Quantum size effects are no longer negligible in Sn nanosystems once the sample size reduces be- low $d \leq \sqrt{\frac{\hbar^{2} \pi^{2}}{2 m_{e} \Delta_{\text {bulk }}}} \backsim 26 \mathrm{~nm}[47]$. In the case of $\mathrm{Sn}$ nanowires, these effects are significant for diameters below $20 \mathrm{~nm}$ [48]. Our calculations using $\mathrm{F}$ (E) only account for the impact of phonon confinement on $\mathrm{T}_{c}$, not QSE. Hence, QSE could slightly offset the electron-phonon coupling enhancement caused by phonon softening [13]. Ultimately, $\mathrm{T}_{c}$ will be enhanced but it will be limited by the interplay between phonon softening and QSE.

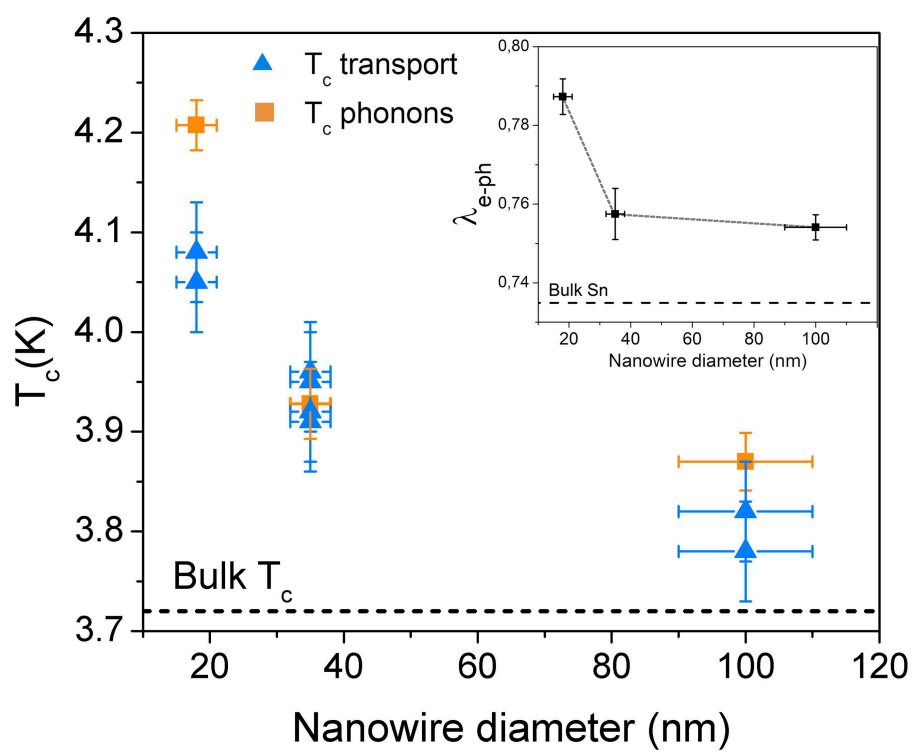

FIG. 7. Comparison between $\mathrm{T}_{c}$ derived from transport and phonon measurements. The inset shows the evolution of the electron-phonon coupling parameter as a function of the nanowire diameter.

The interaction between the nanowires and the $\mathrm{Al}_{2} \mathrm{O}_{3}$ matrix is expected to influence the $\mathrm{T}_{c}$ enhancement. $\mathrm{Al}_{2} \mathrm{O}_{3}$ is harder than $\mathrm{Sn}$ since the vibrational spectrum of $\mathrm{Al}_{2} \mathrm{O}_{3}$ spans up to $120 \mathrm{meV}$ [49], therefore, the $\mathrm{Al}_{2} \mathrm{O}_{3}$ matrix is expected to reduce the number of surface phonon modes as compared to the case of free standing nanowires, limiting the effects of phonon softening without suppressing them completely. This is analogous to the case of nanoparticles capped with a hard layer, the number of surface modes that shifts towards lower energies is smaller than in the case of free particles [50], since the energy of the nanoparticle surface modes is increased by the interaction with the capping layer. Moreover, Bessas et al. measured the phonon density of states of $\mathrm{Bi}_{2} \mathrm{Te}_{3}$ nanowires with a diameter of $56 \mathrm{~nm}$ embedded in $\mathrm{Al}_{2} \mathrm{O}_{3}$ [51]. No significant differences were found when comparing the phonon density of states of the nanowires to the one of bulk $\mathrm{Bi}_{2} \mathrm{Te}_{3}$. Their analysis concluded that the Debye level $\left(\lim _{E \longrightarrow 0} F(E) / E^{2}\right)$ of the nanowires was higher than the Debye level of bulk $\mathrm{Bi}_{2} \mathrm{Te}_{3}$, which caused a decrease of the nanowire sound velocity of $6.5 \%$. They attributed this reduction to confinement due to nanostructuring. In addition, they estimated that only phonons with an energy of $0.12 \mathrm{meV}$ 
will be affected by interactions with the $\mathrm{Al}_{2} \mathrm{O}_{3}$ matrix. This leads to the conclusion that the $\mathrm{Al}_{2} \mathrm{O}_{3}$ matrix does not strongly interact with the $\mathrm{Bi}_{2} \mathrm{Te}_{3}$.

Note that the phonon density of states of Sn and Te are quite similar, both of them show a phonon energy cutoff around $18 \mathrm{meV}$ and the Debye behavior extends only up to $4 \mathrm{meV}$. Moreover, similarly to the case of $\mathrm{Bi}_{2} \mathrm{Te}_{3}$ nanowires, the Debye level of Sn nanowires is higher than the one of bulk Sn. Finally, the number of $\mathrm{Al}_{2} \mathrm{O}_{3}$ phonon modes available to interact with the $\mathrm{Sn}$ phonon modes is small, since the Sn phonon cut-off occurs at $18 \mathrm{meV}$, while the majority of the phonon modes in $\mathrm{Al}_{2} \mathrm{O}_{3}$ occurs above $25 \mathrm{meV}$. These features suggest that there is not a strong interaction between the $\mathrm{Al}_{2} \mathrm{O}_{3}$ matrix and $\mathrm{Sn}$ nanowires and that the differences between the Sn nanowires $\mathrm{F}(\mathrm{E})$ and the bulk $\mathrm{Sn} F(\mathrm{E})$ are a consequence of the nanostructuring.

Phonon softening affects any superconducting nanostructure, regardless of its coupling strength. As mentioned before, $\mathrm{T}_{c}$ of weak-coupling superconductors increases upon size reduction while no impact or even a reduction of $\mathrm{T}_{c}$ is observed in strong coupling materials. Note that, according to theoretical calculations, strongcoupling superconducting nanograins exhibit a larger broadening of single electron levels and a heavier electron mass than weak-coupling superconductors [52]. QSE are inversely proportional to the electron mass, thus making them more relevant in strong-coupling superconductors. Then, QSE could offset or even overcome the effect of phonon softening in strong-coupling materials, resulting in an unaffected value of $\mathrm{T}_{c}$.

In summary, for the first time the phonon density of states of Sn nanowires with diameters of $18 \mathrm{~nm}, 35$ $\mathrm{nm}$ and $100 \mathrm{~nm}$ has been experimentally determined and used to calculate their $\mathrm{T}_{c}$. The correspondence between the critical temperature measured by trans- port measurements and the one calculated from the measured $\mathrm{F}(\mathrm{E})$ suggests that phonon softening plays a significant role in the $\mathrm{T}_{c}$ enhancement, although other effects, in particular electron confinement, might also affect the $T_{c}$ of the smallest nanowires investigated. In addition, our results provide a direct correlation between phonon confinement effects, the modification of the electron-phonon coupling strength $\lambda_{e-p h}$ and the $\mathrm{T}_{c}$ enhancement of a weak-coupling superconductor. Further research on the modifications of $\mathrm{F}(\mathrm{E})$ in both, weak and strong-coupling nanoscale superconductors, as well as the shape of $\alpha^{2}(\mathrm{E})$ will help to understand the specific roles of phonon and electronic confinement. Our work enables the quantification of phonon softening effects, assessing its impact in the modification of the electron-phonon coupling strength at the nanoscale.

\section{ACKNOWLEDGMENTS}

We would like to thanks Jeroen Scheerder and Wout Keijers for their help and assistance during the low temperature measurements. This work was supported by the Research Foundation Flanders (FWO), the Concerted Research Action (GOA/14/007), the Fédération Wallonie-Bruxelles (ARC 13/18-052, Supracryst) and the Fonds de la Recherche Scientifique FNRS under Grant $\mathrm{n}^{\circ}$ T.0006.16 . The authors acknowledge Hercules Stichting (Projects No. AKUL/13/19 and No. AKUL/13/25). D. P. Lozano thanks the FWO for financial support. This research used resources of the Advanced Photon Source, a US Department of Energy (DOE) Office of Science User Facility operated for the DOE Office of Science by Argonne National Laboratory under Contract No. DEAC02-06CH11357.
[1] A. D. Wright, C. Verdi, R. L. Milot, G. E. Eperon, M. A. Pérez-Osorio, H. J. Snaith, F. Giustino, M. B. Johnston and L. M. Herz, Nature Communications 7, 11755 (2016).

[2] T. Wang, Z. Gui, A. Janotti, C. Ni, and P. Karandikar, Phys. Rev. Materials 1, 034601 (2017).

[3] J. Bardeen, L. N. Cooper, and J. R. Schrieffer, Phys. Rev. 108, 1175 (1957).

[4] S. Bose and P. Ayyub, Rep. Prog. Phys. 77116503 (2014).

[5] S. Dubois, A. Michel, J. P. Eymery, J. L. Duvail and L. Piraux, Journal of Materials Research, 14(3), pp. 665671 (1999).

[6] M. He, C. H. Wong, P. L. Tse, Y. Zheng, H. Zhang, F. L. Y. Lam, P. Sheng, X. Hu and R. Lortz, ACS Nano, 7 (5), 41874193 (2013).

[7] S. Bose, A. M. Garca-Garca, M. M. Ugeda, J. D. Urbina, C. H. Michaelis, I. Brihuega and K. Kern, Nature Materials 9, 550554 (2010).

[8] P. B. Allen, Handbook of Superconductivity, (edited by C. P. Poole, Jr., Academic Press), (New York), (1999),
Ch. 9, Sec. G, pp. 478-483.

[9] F. Altomare, A. M. Chang, M. R. Melloch, Y. Hong and Charles W. Tu, Phys. Rev. Lett. 97, 017001 (2006).

[10] M. Tian, J. Wang, J. S. Kurtz, Y. Liu, M. H. W. Chan, T. S. Mayer, and T. E. Mallouk, Phys. Rev. B 71, 104521 (2005).

[11] F.Y. Wu, C. C. Yang, C.M. Wu, C.W. Wang, and W.H. Lia, J. Appl. Phys, 101, 09G111 (2007).

[12] B. Abeles, R. W. Cohen, and G. W. Cullen, Phys. Rev. Lett. 17, 632 (1966).

[13] S. Bose, C. Galande, S. P. Chockalingam, R. Banerjee, P. Raychaudhuri and P. Ayyub, J. Phys.: Condens. Matter 21205702 (2009).

[14] G. C. Tettamanzi, C. I. Pakes, A. Potenza, S. Rubanov, C. H. Marrows and S. Prawer, Nanotechnology 20, 465302, (2009).

[15] P. P. Parshin, M. G. Zemlyanov, G. Kh. Panova, A. A. Shikov, A. A. Naberezhnov, Yu. A. Kumzerov, I. V. Golosovsky, A. S. Ivanov, J. Exp. Theor. Phys. 111, 6 (2010). 
[16] M. D. Croitoru, A. A. Shanenko, and F. M. Peeters Phys. Rev. B 76, 024511 (2007).

[17] M. Strongin, O. F. Kammerer, J. E. Crow, R. D. Parks, D. H. Douglass, Jr., and M. A. Jensen, Phys. Rev. Lett. 21 (18), 1320-1323 (1968).

[18] P. P. Parshin, M. G. Zemlyanov, G. Kh. Panova, A. A. Shikov, Yu. A. Kumzerov, A. A. Naberezhnov, I. Sergueev, W. Crichton, A. I. Chumakov, R. Rffer, J. Exp. Theor. Phys. 114, 3 (2012).

[19] S. Couet, H. Peelaers, M. Trekels, K. Houben, C. Petermann, M. Y. Hu, J. Y. Zhao, W. Bi, E. E. Alp, E. Menéndez, B. Partoens, F. M. Peeters, M. J. Van Bael, A. Vantomme, and K. Temst, Phys. Rev. B 88, 159903(E)(2013).

[20] J. M. Rowell, W. L. McMillan, and W. L. Feldmann, Phys. Rev. B 3, 4065 (1971).

[21] L. Zaraska, E. Kurowska, G. D. Sulka and M. Jaskua, Applied Surface Science 258, 9718 - 9722 (2012).

[22] M. Tian, N. Kumar, S. Xu, J. Wang, J. S. Kurtz, and M. H. W. Chan, Phys. Rev. Lett. 95, 076802 (2005).

[23] L. Lutterotti and P. Scardi, J. Appl. Crys. 23, 246 (1990).

[24] MAUD (Materials Analysis Using Diffraction), http://maud.radiographema.com/

[25] B. D. Cullity, Elements of X-ray Diffraction, (AddisonWesley Publishing Company, Inc.), (Reading, Massachusetts, USA), (1956), Page 262.

[26] See Supplemental Material at [URL will be inserted by publisher] For details on the transport measurements and the phonon density of states when the X-ray beam is parallel and perpendicular to the nanowires axis.

[27] L. Jankovič, D. Gournis, P. N. Trikalitis, I. Arfaoui, T. Cren, P. Rudolf, M. Sage, T. T. M. Palstra, Bart Kooi, J. De Hosson, Mi. A. Karakassides, K. Dimos, A. Moukarika, and T. Bakas, Nano Lett., 2006, 6, 1131 (2006).

[28] Y. Zhang, C. Ho Wong, J. Shen, S. T. Sze, B. Zhang, H. Zhang, Y. Dong, H. Xu, Z. Yan, Y. Li, X. Hu and R. Lortz, Scientific Reports 6, 32963 (2016).

[29] C. Kittel "Introduction to Solid State Physics", John Wiley \& Sons, Inc., New York (1986).

[30] M. Tian, J. Wang, J. Snyder, J. Kurtz, Y. Liu, P. Schiffer, T. E. Mallouk, and M. H. W. Chan, Appl. Phys. Lett. 83, 1620 (2003).

[31] V. V. Moshchalkov, J. Fritzsche Nanostructured Superconductors, Chapter 2 - Individual nanostructures. Nanostructured Superconductors. Ed. by V. V. Moshchalkov. First Edition. Singapore: World Scientific Publishing (2011).

[32] V. V. Moshchalkov, L. Gielen, C. Strunk, R. Jonckheere, X. Qiu, C. Van Haesendonck, and Y. Bruynseraede, Nature 373, pp. 319321 (1995).
[33] M. Tinkham. Introduction to Superconductivity. Ed. by M. Tinkham. Second Edition. New York: McGrawHill, 1975.

[34] S. Bose, P. Raychaudhuri, R. Banerjee, and P. Ayyub, Phys. Rev. B 74, 224502 (2006).

[35] L. Fàbrega, A Camón, I. Fernández-Martínez, J. Sesé, M. Parra-Borderías, O. Gil, R González-Arrabal, J. L. Costa-Krämer and F. Briones, Superconductor Science and Technology, 24, 7 (2011).

[36] A. Chumakov and W. Sturhahn, Hyperfine Interactions 123, 781 (1999).

[37] R. Röhlsberger, J. Phys. Condens. Matter 13, 7659 (2001).

[38] B. M. Leu, M. Sturza, M. Y. Hu, D. Gosztola, V. Baran, T. F. Fässler and E. E. Alp, Phys. Rev. B 90, 104304 (2014).

[39] W. Sturhahn, J. Phys. Condens. Matter 16 S497S530 (2004).

[40] W. Sturhahn, Hyperfine Interactions 125, 149 (2000).

[41] V. G. Kohn, A. I. Chumakov and R. Rüffer, Phys. Rev. B 58, 8437 (1998).

[42] K. Houben, PhD Thesis, Vibrational and superconducting properties of Sn nanostructures and hybrid assemblies, KU Leuven (2015).

[43] P. B. Allen and R. C. Dynes Phys. Rev. B 12, 905 (1975).

[44] R.C. Dynes, Solid State Commun. 10, 7, 615 (1972).

[45] S. Stankov, R. Röhlsberger, T. Ślęzak, M. Sladecek, B. Sepiol, G. Vogl, A. I. Chumakov, R. Rüffer, N. Spiridis, J. Łażewski, K. Parliński, and J. Korecki, Phys. Rev. Lett. 99, 185501 (2007).

[46] K. Houben, S. Couet, M. Trekels, E. Menéndez, T. Peissker, J. W. Seo, M. Y. Hu, J. Y. Zhao, E. E. Alp, S. Roelants, B. Partoens, M. V. Miloševic, F. M. Peeters, D. Bessas, S. A. Brown, A. Vantomme, K. Temst, and M.J. Van Bael, Phys. Rev. B 95, 155413 (2017).

[47] F. M. Peeters, A. A. Shanenko, and M. D. Croitoru. Handbook of Nanophysics: Principles and Methods, Chapter 9 - Nanoscale Superconductivity. Ed. by K. D. Sattler. First. Boca Raton: CRC Press, pp. 9.19.32 (2010).

[48] A. A. Shanenko, M. D. Croitoru, M. Zgirski, F. M. Peeters and K. Arutyunov, Phys. Rev. B 74, 052502 (2006).

[49] S. Davis and G. Gutiérrez, Journal of Physics: Condensed Matter 23, 49, p. 495401 (2011).

[50] A. Tamura, Z. Phys. D 26 (suppl.) 240 (1993).

[51] D. Bessas, W. Töllner, Z. Aabdin, N. Peranio, I. Sergueev, H. Wille, O. Eibl, K. Nielsch and R. P. Hermann, Nanoscale, 5, 10629-10635 (2013).

[52] M. D. Croitoru, A. A. Shanenko, A. Vagov, M. V. Milošević, V. M. Axt and F. M. Peeters, Scientific Reports 5, 16515 (2015). 